\title{
Review Article \\ Recent Advances of the NLRP3 Inflammasome in Central Nervous System Disorders
}

\author{
Keren Zhou, ${ }^{1,2}$ Ligen Shi, ${ }^{1,2}$ Yan Wang, ${ }^{1,2}$ Sheng Chen,,2 and Jianmin Zhang ${ }^{1,2}$ \\ ${ }^{1}$ Department of Neurosurgery, Second Affiliated Hospital, School of Medicine, Zhejiang University, Hangzhou, Zhejiang, China \\ ${ }^{2}$ Brain Research Institute, Zhejiang University, Hangzhou, Zhejiang, China \\ Correspondence should be addressed to Sheng Chen; saintchan@sina.com
}

Received 15 June 2016; Accepted 2 August 2016

Academic Editor: Menaka C. Thounaojam

Copyright (c) 2016 Keren Zhou et al. This is an open access article distributed under the Creative Commons Attribution License, which permits unrestricted use, distribution, and reproduction in any medium, provided the original work is properly cited.

\begin{abstract}
Inflammasomes are multiprotein complexes that trigger the activation of caspases-1 and subsequently the maturation of proinflammatory cytokines interleukin- $1 \beta$ and interleukin-18. These cytokines play a critical role in mediating inflammation and innate immunity response. Among various inflammasome complexes, the NLRP3 inflammasome is the best characterized, which has been demonstrated as a crucial role in various diseases. Here, we review recently described mechanisms that are involved in the activation and regulation of NLRP3 inflammasome. In addition, we summarize the recent researches on the role of NLRP3 inflammasome in central nervous system (CNS) diseases, including traumatic brain injury, ischemic stroke and hemorrhagic stroke, brain tumor, neurodegenerative diseases, and other CNS diseases. In conclusion, the NLRP3 inflammasome may be a promising therapeutic target for these CNS diseases.
\end{abstract}

\section{Introduction}

In the central nervous system (CNS), the innate immune response plays a significant role in the pathology after tissue damage or pathogen invasion. This process is known as neuroinflammation and is characterized by the activation of the microglia and astrocyte population [1]. It is known that several cell types in the brain express specialized pattern recognition receptors (PRRs) such as membranebound Toll-like receptors (TLRs) and cytosolic NOD-like receptors (NLRs). The NOD-like receptors are a class of cytosolic sensors or receptors that respond to a variety of pathogen-associated molecular patterns (PAMPs) linked to various microbes and damage-associated molecular patterns (DAMPs) produced during tissue-based injury [2]

One of the most extensively studied classes of NLRs is the inflammasome-forming NLRs including NLRP1, NLRP3, NLRC4, NLRC5, NLRP6, NLRP7, and NLRP12 as well as the non-NLR inflammasome receptor known as AIM2. Among them, NLRP3 is the best characterized. The NLRP3 inflammasome is composed of NLRP3, apoptosis-associated specklike (ASC) adapter protein, and the downstream effector enzyme (procaspase-1) [3]. When stimulated by PAMP or
DAMP, NLR forms a protein complex known as the inflammasome through the combination of the adaptor protein ASC [4]. This initiates the cleavage of procaspase-1 into the active and mature form of caspase-1 [5]. Subsequently, active caspase- 1 converts the inactive pro-IL- $1 \beta$ and pro-IL18 into their active and secreted forms: IL- $1 \beta$ and IL-18. These cytokines initiate or amplify diverse downstream signaling pathways and drive proinflammatory responses, leading to cellular damage, such as autophagy and pyroptosis $[6,7]$.

Recently, increasing attention is being paid to the role of the NLRP3 inflammasome in the central nervous system (CNS). The NLRP3 inflammasome plays a pathogenic role in neuroinflammatory diseases. Here, we review described mechanisms that have been proposed to be involved in the activation and regulation of NLRP3 inflammasome and further explore the role of NLRP3 inflammasome in several CNS diseases.

\section{The Activation and Regulation of NLRP3 Inflammasome (Figure 1)}

To date, it has been demonstrated that the activation of the NLRP3 inflammasome appears to occur by two signals. 


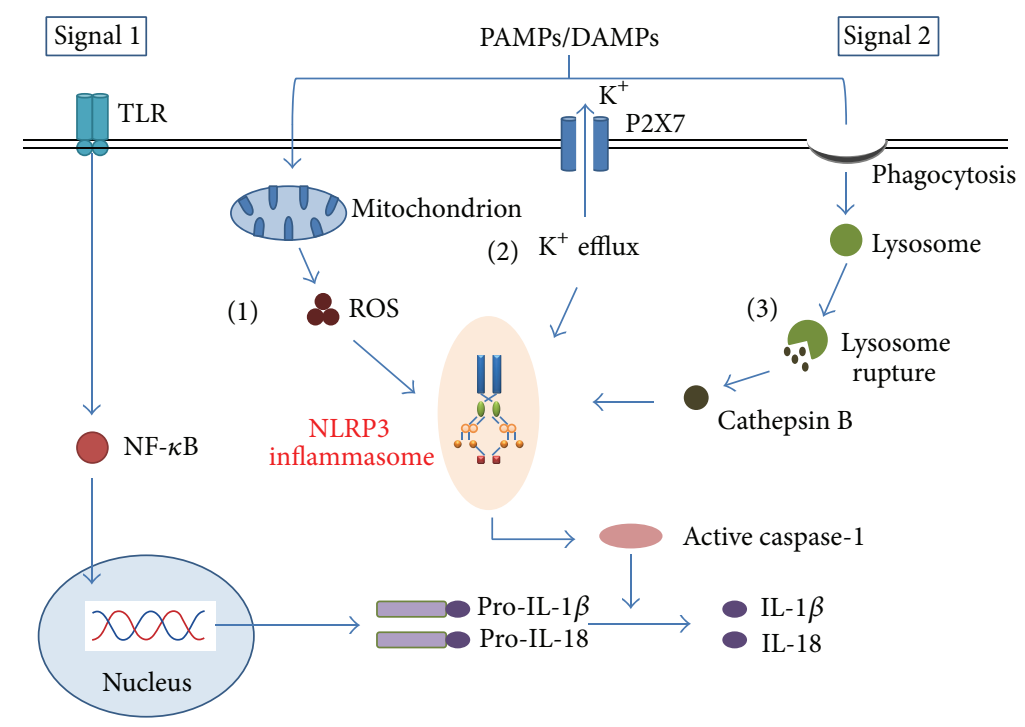

FIgURE 1: Models of NLRP3 inflammasome activation. Signal 1 activates the Toll-like receptor (TLR)/NF- $\kappa$ B pathway, leading to the transcription of pro-IL-1 $\beta$ and pro-IL-18. Signal 2 is mediated by PAMPs or DAMPs stimulation and promotes the assembly of NLRP3 inflammasome complex. Three main mechanisms of the NLRP3 inflammasome activation have been proposed. (1) Stimuli can trigger the production of mitochondrial ROS and then induce the NLRP3 inflammasome activity. (2) Extracellular ATP or bacterial toxins can induce $\mathrm{K}^{+}$efflux through the P2X purinergic receptor 7 (P2X7), which leads to the activation of the NLRP3 inflammasome. (3) The phagocytosis of specific crystalline can cause lysosomal rupture and induce the release of lysosomal contents cathepsin $\mathrm{B}$, which contributes to the activation and assembly of the NLRP3 inflammasome. Consequently, these trigger the cleavage of procaspase-1 into its active and mature form caspase-1, which leads to the production of the mature IL-1 $\beta$ and IL-18.

The initial priming signal, induced by the Toll-like receptor (TLR)/nuclear factor NF- $\kappa$ B pathway, affects NLRP3 at the transcriptional level and also serves to trigger posttranslational modifications of inflammasome components $[8,9]$. The second signal triggers assembly of the NLRP3 inflammasome complex $[10,11]$. The NLRP3 inflammasome complex can be activated by both exogenous (including infection, tissue damage, and metabolic dysregulation) [12] and endogenous molecules such as extracellular ATP, hyaluronan, A $\beta$ fibrils, and uric acid crystals [13].

For a large number and diversity of NLRP3 inflammasome stimuli, it seems unlikely that they all bind to the NLRP3 structure to form the NLRP3 inflammasome. So far, there have been three mainly mechanisms regarding activation of the NLRP3 inflammasome, including the generation of reactive oxygen species (ROS), the efflux of potassium, and the rupture of lysosomal $[14,15]$.

2.1. Reactive Oxygen Species (ROS). ROS, mainly associated with the normal or malfunctioning mitochondria, has been proved to play a significant role in the activation of NLRP3 inflammasome [16]. Numerous NLRP3 inflammasome activators are known to trigger mitochondrial ROS production.

A recent study has shown that the thioredoxin-interacting protein (TXNIP) is a ROS-sensitive regulator of the activation of NLRP3 inflammasome [17]. The binding of TXNIP to NLRP3 leads to the activation of NLRP3, the recruitment of ASC and procaspase-1, and the formation of the active NLRP3 inflammasome complex [18, 19]. In addition, several researches suggest that the damage to NADPH oxidase by mitochondrial ROS can activate the inflammasome [20,
21]. Other studies suggest that NADPH oxidase and the production of ROS are dispensable for NLRP3 inflammasome activation, but crucial for IL- $1 \beta$ secretion $[15,22]$. Moreover, it has been demonstrated that the mitochondria-targeted antioxidant Mito-TEMPO, which targets mitochondrial ROS, can inhibit the activation of inflammasome and subsequently reduce the secretion of IL- $1 \beta$ and IL-18 $[23,24]$.

However, ROS activation is not an absolute requirement for activation of all NLRP3 inflammasomes [25]. Some studies implicate that mitochondrial ROS mediates the upregulation of NLRP3 and pro-IL-1 $\beta$ transcription rather than the NLRP3 inflammasome activation [26]. Therefore, more studies are required to understand the precise role of ROS in regulating NLRP3 inflammasome activation.

2.2. $K^{+}$Efflux. Another fully studied mechanism of NLRP3 inflammasome activation is the decrease in the intracellular $\mathrm{K}^{+}$. Several mechanisms underlying the efflux of $\mathrm{K}^{+}$ have been proposed. For example, high extracellular ATP concentrations can reduce intracellular $\mathrm{K}^{+}$concentrations by activating the $\mathrm{P} 2 \mathrm{X}$ purinergic receptor 7 (P2X7), which is considered an important signaling pathway to activate NLRP3 inflammasomes [27, 28]. Furthermore, a reduction in intracellular $\mathrm{K}^{+}$levels was found to be essential for NLRP3 inflammasome activation when triggered by bacterial infection, MSU crystals, and pore-forming toxins [29, 30]. Another study indicated that inflammasome activation in response to many NLRP3 activators was effectively inhibited by $\mathrm{K}^{+}$channel inhibitor glibenclamide [13]. Recently, the reduction in intracellular $\mathrm{K}^{+}$concentration is thought to be a common pathway for NLRP3 inflammasome complex 
activation. However, the mechanisms of how low cytoplasmic $\mathrm{K}^{+}$concentration activates inflammasome activation are needed to further study.

2.3. Rupture of Lysosome. It has also been widely accepted that the disruption of the lysosomal membrane can result in NLRP3 activation. lysosome destabilization, caused by the phagocytosis of specific crystalline and particulate matter, leads to the release of lysosomal contents [31]. Cathepsin $\mathrm{B}$, as one of the lysosomal contents, has been proved to activate the NLRP3 inflammasome. Cathepsin B inhibitor CA-074-Me was found to partially inhibit NLRP3 activation $[32,33]$. Besides the mechanisms of activation by endogenous crystalline, the role of environmentally derived crystals such as asbestos, silica, and aluminum salts has also been proved in NLRP3 inflammasome activation [34]. In addition, to understand how lysosomal rupture leads to NLRP3 activation, a recent study found that the TAK1-JNK pathway, a MAPK signaling pathway, was activated through lysosome rupture and that this activation was necessary for the complete activation of the NLRP3 inflammasome [35]. Thus, the lysosome plays an essential role in the activation of the NLRP3 inflammasome. Further understanding of the mechanism is needed in future.

2.4. Other Activators. Despite the former three mechanisms regarding activation of the NLRP3, it is becoming increasingly clear that one signal alone is insufficient to induce inflammasome activation [16]. Several other pathways have been revealed to explain the mechanisms by which diverse stimuli activate the NLRP3 inflammasome complex. Recently, it has been reported that $\mathrm{Ca} 2+$ mobilization mediated mitochondrial damage and dysfunction can also activate the NLRP3 inflammasome [36, 37]. In addition, mitochondria-associated cardiolipin is required for recruitment and activation of the NLRP3 inflammasome [38]. Moreover, some studies elucidated the contribution of the mitochondrial antiviral signaling protein (MAVS) in NLRP3 inflammasome activation [39, 40]. It has also been reported that infection with RNA virus initiates assembly of the RIP1-RIP3 complex, which promotes activation of the GTPase DRP1 and its translocation to mitochondria to drive mitochondrial damage and activation of the NLRP3 inflammasome [41].

2.5. Negative Regulation. Despite the multiple positive regulation contributed to the activation of NLRP3 inflammasome, negative regulation is also necessary to maintain appropriate induction of inflammasome function.

Recent studies have indicated that autophagy can act as a negative regulator of NLRP3 inflammasome activation by removing sources of endogenous NLRP3 inflammasome agonists $[42,43]$, suppressing of IL- $1 \beta$ secretion [44], and degrading inflammasome components, such as NLRP3 and ASC [45, 46]. And also inhibition of autophagy by 3methyladenine (3-MA) can promote the activation of the NLRP3 inflammasome [47]. Additionally, nitric oxide (NO) downregulates NLRP3 activation through enhancing the removal of the dysfunctional mitochondria and preventing assembly of the inflammasome $[48,49]$. Moreover, type I IFNs inhibit the NLRP3 inflammasome in both the priming signal and the activation signal [50]. Other forms of negative regulation, such as the roles of microRNAs and bacterial and viral mechanisms, have been detailed in recent reviews $[8,51]$.

Considering the recent findings of activation and regulation of the NLRP3 inflammasome, further understanding of these molecular mechanisms and signal pathways may be helpful in designing potential therapeutics to prevent inflammatory diseases associated with the NLRP3 inflammasome.

\section{NLRP3 Inflammasome and CNS Diseases}

Recently, an increasing number of studies have been investigating the underlying role of NLRP3 inflammasome in the central nervous system. The NLRP3 inflammasome has been proved to express in diversity of cells such as microglia, astrocyte, neuron, and endothelial cell [52-55] and in different kinds of diseases such as traumatic brain injury, stroke, brain tumor, neurodegenerative disease, and others.

3.1. NLRP3 Inflammasome and Traumatic Brain Injury. Traumatic brain injury (TBI), caused by physical force to the brain tissue, initiates a primary insult and secondary cascade of events. The primary insult results in direct neuronal loss and necrotic death, which is then followed by a wave of injury cascades including excitotoxicity, oxidative stress, mitochondrial dysfunction, blood-brain barrier disruption, and inflammation [56]. Numerous studies laid emphasis on the role of inflammatory response among the components of the secondary brain injury $[57,58]$.

The significant increasing of IL- $1 \beta$ and IL-18 after experimental TBI has been demonstrated [59]. Recently, a study found that TBI could induce assembly of NLRP3 inflammasome complex, expression of ASC, activation of caspase1 , and processing of IL- $1 \beta$ and IL-18. It may be possible that NLRP3 inflammasome is a promising therapeutic target for patients with TBI [60]. Similar to NLRP3, NLRP1 has also been proved as an important component of the innate inflammatory response after TBI. TBI induced assembly of the NLRP1 inflammasome, cleavage of X-linked inhibitor of apoptosis protein (XIAP), activation of caspase-1, and processing of IL-1 $\beta$ [61]. Another study showed that TBI patients with higher levels of NLRP1 in the cerebrospinal fluid (CSF) had a worse outcome than that with a lower expression of NLRP1 [62]. NLRP1 and NLRP3 may work together to play a role in the inflammation after TBI and serve as candidate therapeutic targets in TBI [4]. However, the relationship between those two inflammasomes needs further study.

Although the studies indicated a correlation between enhanced inflammasome expression and TBI pathology, the potential functional role that inflammasome may play in TBI remains to be directly demonstrated. Therefore, inflammatory response, especially in the postinjury period of TBI, is an important therapeutic target for reducing the neurological dysfunction and improving the outcome. 
3.2. NLRP3 Inflammasome and Ischemia Stroke. Stroke is regarded as a severe disorder with high mortality and long-term disability. Clinically, stroke can be classified into ischemic stroke and hemorrhagic stroke. Ischemic stroke commonly accounts for approximately $80 \%$ of all stroke cases while hemorrhagic stroke accounts for approximately $20 \%$ [63]. The mechanisms responsible for ischemic strokeinduced neuronal cell death include bioenergetic failure, oxidative stress, excitotoxicity, apoptosis, and inflammatory process [64-66].

Recently, the increasing number of evidences has indicated that inflammatory mechanism fundamental to the innate immune system may contribute to the death of neuronal and glial cell during cerebral ischemia [67]. The NLRP3 inflammasome has been proved to play an important role in detecting cellular damage and mediating inflammatory responses to tissue injury during ischemic stroke. Hence, targeting pathways upstream and downstream of NLRP3 inflammasome signaling may offer substantial promise in developing new therapeutics for stroke [68].

A study found the neuroprotective effects of intravenous immunoglobulin (IVIg), which could significantly reduce the levels of NLRP3 inflammasome proteins as well as IL$1 \beta$ and IL-18 during simulated ischemia in vitro and in a mouse model of focal ischemic stroke [69]. Another study showed that intermittent fasting (IF) could contribute to reducing expression of NLRP1 and NLRP3 inflammasome and the precursors of IL-1 $\beta$ and IL-18 in a mouse model of focal ischemic stroke by suppressing the activation of NF- $\kappa \mathrm{B}$ and MAPK pathway [70]. More evidence showed that, in neuronal cells, oxygen-glucose deprivation (OGD) could induce the accumulation of dsRNA to prime the NLRP3 and proinflammatory cytokines production, which were involved in the inflammatory progression and injuries of cerebral ischemia [71]. These suggest that targeting NF- $\kappa \mathrm{B}$ pathway and transcriptional level may provide a therapeutic effect on inflammasome expression and activity during cerebral ischemia. Recently, the research targeting TXNIP/NLRP3 inflammasome activation is also a hotspot. A study indicated that Umbelliferone (UMB) suppressed the inflammatory cytokines production through the inhibition of TXNIP/NLRP3 inflammasome activation [72]. Another study showed that curcumin inhibited TXNIP/NLRP3 inflammasome activation by suppressing endoplasmic reticulum stress and thereby protected neuronal cell survival from glutamate neurotoxicity after ischemic insult [73]. In addition, one study demonstrated that pharmacological inhibition by using resveratrol or genetic deletion of TXNIP attenuated brain infarction and neurological outcome in mice embolic mode via restoring redoxbalance and inhibition of TXNIP-NLRP3 inflammasome activation [74]. Moreover, there are also researches targeting inflammasome components NLRPs, ASC, and caspase-1. One study demonstrated that estrogen and progesterone could regulate ASC and NLRP3 at the protein level and reduce the expression of inflammasome components in the transient focal rat ischemic model [53]. In addition, Bruton's tyrosine kinase (BTK) regulated activation of the NLRP3 inflammasome by interacting with NLRP3 and ASC [75]. Another study found that A151, a synthetic oligodeoxynucleotide, attenuated ischemic brain injury by reducing the maturation of caspase- 1 and IL-1 $\beta$ and the production of NLRP3 [76].

Therefore, numerous studies have focused on the expression, activity, and products of NLRP3 inflammasome, which may discover potential therapeutics for ischemia stroke. However, there are still many underlying mechanisms of the inflammasome remained to investigate.

3.3. NLRP3 Inflammasome and Hemorrhagic Stroke. Spontaneous intracerebral hemorrhage (ICH), as one type of hemorrhagic stroke, is also a devastating disease with high morbidity and mortality [77]. The mechanisms responsible for neurological dysfunction after ICH include hematoma formation, brain edema, inflammation, and microglia activation [78-81]. Accumulating evidence indicates that inflammatory mechanism, especially the role of NLRP3 inflammasome, is involved in the pathophysiology of ICH [82].

In a mouse model of $\mathrm{ICH}, \mathrm{Ma}$ et al. found the role of NLRP3 inflammasome in contributing to ICH-induced inflammatory activation. The mPTP inhibitor (TRO-19622) and mitochondria ROS scavenger (Mito-TEMPO) were used in the study to indicate the mechanism of mitochondria ROS in NLRP3 inflammasome activation [24]. The inhibition of $\mathrm{P} 2 \mathrm{X} 7 \mathrm{R}$ pathway by using blue brilliant $\mathrm{G}$ (BBG) could be a potential therapeutic target for secondary brain injury after ICH [83]. Additionally, a recent study found that a recombinant adenovirus encoding NLRP3 RNAi attenuated inflammation in ICH [84]. Moreover, another study identified miR-223 suppressed NLRP3 production by directly binding to its $3^{\prime}$ UTR, which reduced neuronal inflammation and improved neuronal function after ICH [85]. Recent studies have revealed new information on the NLRP3 inflammasome during ICH, and the NLRP3 inflammasome may be a promising therapeutic target in ICH patients.

Subarachnoid hemorrhage (SAH), as another type of hemorrhagic stroke, is associated with a high mortality and morbidity [86, 87]. Recently, increasing evidence has emphasized the role of early brain injury (EBI) associated with the poor outcome of SAH patients [88-91]. The underlying mechanisms include a reduction in cerebral blood flow, increased intracranial pressure, oxidative stress, apoptosis, and inflammation [92]. NLRP3 inflammasome has been proved a key component of inflammatory response in the pathophysiology of SAH.

A recent study made the observation that treatment with minocycline in a rat SAH model could inhibit NLRP3 inflammasome activation and attenuate brain edema in EBI after SAH. This effect may be associated with the reduction of mitochondrial ROS [93]. Another study demonstrated the downregulating role of hydrogen-rich saline (HS) treatment in the activation of NLRP3 inflammasome via inhibiting NF$\kappa \mathrm{B}$ pathway after $\mathrm{SAH}[94]$. In addition, the neuroprotection role of melatonin treatment has been proved in the EBI following SAH by inhibiting NLRP3 inflammasome activation and NLRP3-associated apoptosis [95].

An increasing number of studies focus on the role of NLRP3 inflammasome in inflammatory response after SAH. 
These studies may provide a promising therapeutic choice for patients with SAH.

3.4. NLRP3 Inflammasome and Brain Tumor. In recent years, the important role of NLRP3 inflammasome in carcinogenesis and tumor progression has been reported. A number of endogenous and exogenous stimuli can behave as tumor promoters by inducing chronic inflammation and consequently provide signals for inflammasome activation in cancer [96]. And NLRP3 inflammasome is also reported to suppress the function of natural killer cell in controlling carcinogenesis and metastases [97]. Moreover, NLRP3 inflammasome plays a significant role in tumor control by recruiting neutrophils, which may provide a prognostic marker and promising therapeutic target in cancer patients [98].

In CNS, malignant glioma is the most common primary brain tumors with poor prognosis. Increasing evidence indicates the crucial role of innate immunity and chronic inflammation in carcinogenesis and tumor progression [99101]. And the role of NLRP3 inflammasome in glioma has also been described. NLRP3 inflammasome can active in glioblastoma multiforme (GBM) cells constitutively [102]. NLRP3 inflammasome can also be activated by different signals in different types of cells $[54,55,103]$. Moreover, by using a mouse glioblastoma model, it has been proved that the inhibition of NLRP3 can reduce tumor growth and prolong the survival of mouse following IR treatment. They demonstrated that NLRP3 inflammasome was a molecular link between brain aging and progression of glioma and radiotherapy resistance [104].

The NLRP3 gene signature may serve as a promising biomarker in glioma patients. However, the potential mechanism of NLRP 3 inflammasome in the progress of brain tumor has not been totally understood. More studies are needed in understanding the character of inflammasome and exploring therapeutic potential in brain cancer.

3.5. NLRP3 Inflammasome and Neurodegenerative Diseases. Recent researches have indicated that innate immune activation and neuroinflammation may be involved in various neurodegenerative diseases, such as Alzheimer's disease (AD) and Parkinson's disease (PD). There is increasing evidence that sustained inflammatory responses may contribute to disease progression. It can not only be a consequence but also be a trigger of pathology [105]. Neuroinflammatory cascades rely on the activation of NLRP3 inflammasome, which has been proved crucial in neurodegenerative diseases.

$\mathrm{AD}$ is the most prevalent form of dementia. Extensive evidence has indicated that IL-1 $\beta$ and IL-18 may contribute to the pathogenesis of $\mathrm{AD}$ and cause cognitive impairment $[106,107]$. The pathogenesis of $\mathrm{AD}$ involves extracellular accumulation of amyloid- $\beta(A \beta)$ in senile plaques [108]. It has been demonstrated that the toxicity of $A \beta$ can activate NLRP3 inflammasome, process IL-1 $\beta$ and IL-18, and eventually induce $\mathrm{AD}$ pathology and tissue damage. Moreover, in $\mathrm{AD}$ transgenic mouse model, the inhibition of NLRP3 can largely protect memory loss and decrease $A \beta$ deposition, which provides a possibility of AD therapy by targeting NLRP3 inflammasome [109].
$\mathrm{PD}$ is another common degenerative disease with two main pathological hallmarks: progressive loss of dopaminergic neurons in substantia nigra and accumulation of Lewy body in neurons [110]. Lewy body is mainly composed of the presynaptic protein $\alpha$-synuclein ( $\alpha$-syn), which has been proved to be a significant role in NLRP3 inflammasome activation and PD pathogenesis [111, 112]. And it also provides a therapeutic possibility for PD.

In addition, numerous researches have revealed the role of NLRP3 inflammasome in many other neurodegenerative diseases such as frontotemporal dementia, amyotrophic lateral sclerosis (ALS), Huntington's disease, and multiple sclerosis (MS) $[105,113]$. The innate immune activation could be an early cause in neurodegenerative diseases and this indicates that anti-inflammatory therapies could be a promising treatment approach. Although the inhibition of inflammation may not alter the underlying cause of disease, it may reduce the production of factors involved in neurotoxicity and consequently result in clinical benefits.

3.6. NLRP3 Inflammasome and Other CNS Diseases. It has been widely accepted that neuroinflammation is involved in the epileptogenesis by promoting neuronal excitability and decreasing seizure threshold [114]. The role of the NLRP3 inflammasome in status epilepticus (SE), one of the most serious types of epilepsy, has been proved. In SE rat model, the expression of NLRP3 inflammasome was promoted, which could activate caspase-1 signaling and contribute to neuroinflammation and epileptogenesis. The inhibition of NLRP3 inflammasome may play a neuroprotective role against neuroinflammation and neuronal damage followed by SE [115].

In addition, increasing evidence shows that the NLRP3 inflammasome is also involved in microbial infections in CNS. Microglial cells constitute the first defense line of the CNS against microbial invasion [116]. A study of Jamilloux et al. found that microglial cells detected that legionella pneumophila could lead to the activation of inflammasome [117]. And another study showed that NLRP3 knockout mice infected with pneumococcal meningitis could present decreased scores of disease severity and brain inflammation [118]. Moreover, another study found the expression of NLRP 3 and IL- $1 \beta$ changed in the brain of avian influenza virus H9N2 infected mice. This study indicated the role of NLRP3 inflammasome in host response to influenza virus infection and the outcome of pathological injury and clinical manifestation [119]. In general, inhibiting inflammasome activation might be a promising target for microbial infection diseases. Besides, many other CNS disorders, such as prion diseases, experimental autoimmune encephalomyelitis (EAE), are associated with the activation of NLRP3 inflammasome $[120,121]$. Further studies are aimed at providing new therapeutic choices for all these disorders.

\section{Conclusion}

In this review, we have elaborated on the mechanisms involved in the activation and regulation of NLRP3 inflammasome. In addition, we collected the recent researches on 
the role of NLRP3 inflammasome in CNS diseases. The exact molecular mechanisms on the assembly, activation, and regulation of NLRP3 inflammasome are required to be further examined, which are very important for NLRP3 inflammasome to be as a novel therapeutic strategy in CNS disorders.

\section{Competing Interests}

The authors declare that there are no competing interests regarding the publication of this paper.

\section{Acknowledgments}

This work was supported by National Natural Science Foundation of China (81500992), Natural Science Foundation of Zhejiang Province (LQ16H090002), and Medical and Health Key Project of Zhejiang Province (2016RCA015).

\section{References}

[1] A. Aguzzi, B. A. Barres, and M. L. Bennett, "Microglia: scapegoat, saboteur, or something else?” Science, vol. 339, no. 6116, pp. 156-161, 2013.

[2] B. K. Davis, H. Wen, and J. P.-Y. Ting, "The Inflammasome NLRs in immunity, inflammation, and associated diseases," Annual Review of Immunology, vol. 29, pp. 707-735, 2011.

[3] F. Martinon, K. Burns, and J. Tschopp, “The Inflammasome: a molecular platform triggering activation of inflammatory caspases and processing of proIL- $\beta$," Molecular Cell, vol. 10, no. 2, pp. 417-426, 2002.

[4] L. C. Freeman and J. P.-Y. Ting, "The pathogenic role of the inflammasome in neurodegenerative diseases," Journal of Neurochemistry, vol. 136, pp. 29-38, 2016.

[5] J. G. Walsh, D. A. Muruve, and C. Power, "Inflammasomes in the CNS," Nature Reviews Neuroscience, vol. 15, no. 2, pp. 84-97, 2014.

[6] L. Niu, S. Zhang, J. Wu, L. Chen, and Y. Wang, "Upregulation of NLRP3 inflammasome in the tears and ocular surface of dry eye patients," PLoS ONE, vol. 10, no. 5, Article ID e0126277, 2015.

[7] K. Schroder and J. Tschopp, “The Inflammasomes," Cell, vol. 140, no. 6, pp. 821-832, 2010.

[8] F. S. Sutterwala, S. Haasken, and S. L. Cassel, "Mechanism of NLRP3 inflammasome activation," Annals of the New York Academy of Sciences, vol. 1319, no. 1, pp. 82-95, 2014.

[9] V. Hornung and E. Latz, "Critical functions of priming and lysosomal damage for NLRP3 activation," European Journal of Immunology, vol. 40, no. 3, pp. 620-623, 2010.

[10] S. K. Vanaja, V. A. K. Rathinam, and K. A. Fitzgerald, "Mechanisms of inflammasome activation: recent advances and novel insights," Trends in Cell Biology, vol. 25, no. 5, pp. 308-315, 2015.

[11] F. Martinon, A. Mayor, and J. Tschopp, "The inflammasomes: guardians of the body," Annual Review of Immunology, vol. 27, pp. 229-265, 2009.

[12] Y. Koizumi, C. Toma, N. Higa, T. Nohara, N. Nakasone, and T. Suzuki, "Inflammasome activation via intracellular NLRs triggered by bacterial infection," Cellular Microbiology, vol. 14, no. 2, pp. 149-154, 2012.
[13] M. Lamkanfi and V. M. Dixit, "Inflammasomes: guardians of cytosolic sanctity," Immunological Reviews, vol. 227, no. 1, pp. 95-105, 2009.

[14] E. Jo, J. K. Kim, D. Shin, and C. Sasakawa, "Molecular mechanisms regulating NLRP3 inflammasome activation," Cellular and Molecular Immunology, vol. 13, no. 2, pp. 148-159, 2015.

[15] J. M. Abais, M. Xia, Y. Zhang, K. M. Boini, and P.-L. Li, "Redox regulation of NLRP3 inflammasomes: ROS as trigger or effector?” Antioxidants \& Redox Signaling, vol. 22, no. 13, pp. 1111-1129, 2015.

[16] O. Gross, C. J. Thomas, G. Guarda, and J. Tschopp, "The inflammasome: an integrated view," Immunological Reviews, vol. 243, no. 1, pp. 136-151, 2011.

[17] R. Zhou, A. Tardivel, B. Thorens, I. Choi, and J. Tschopp, "Thioredoxin-interacting protein links oxidative stress to inflammasome activation," Nature Immunology, vol. 11, no. 2, pp. 136-140, 2010.

[18] I. N. Mohamed, S. S. Hafez, A. Fairaq, A. Ergul, J. D. Imig, and A. B. El-Remessy, "Thioredoxin-interacting protein is required for endothelial NLRP3 inflammasome activation and cell death in a rat model of high-fat diet," Diabetologia, vol. 57, no. 2, pp. 413-423, 2014.

[19] X. Zhang, J.-H. Zhang, X.-Y. Chen et al., "Reactive oxygen species-induced TXNIP drives fructose-mediated hepatic inflammation and lipid accumulation through NLRP3 inflammasome activatio," Antioxidants and Redox Signaling, vol. 22, no. 10, pp. 848-870, 2015.

[20] E. A. Bordt and B. M. Polster, "NADPH oxidase- and mitochondria-derived reactive oxygen species in proinflammatory microglial activation: a bipartisan affair?" Free Radical Biology and Medicine, vol. 76, no. 1, pp. 34-46, 2014.

[21] D. D. Crane, T. J. Bauler, T. D. Wehrly, and C. M. Bosio, "Mitochondrial ROS potentiates indirect activation of the AIM2 inflammasome," Frontiers in Microbiology, vol. 5, article 438, 2014.

[22] M. L. Gabelloni, F. Sabbione, C. Jancic et al., "NADPH oxidase derived reactive oxygen species are involved in human neutrophil IL- $\beta$ secretion but not in inflammasome activation," European Journal of Immunology, vol. 43, no. 12, pp. 3324-3335, 2013.

[23] J. Trnka, F. H. Blaikie, A. Logan, R. A. J. Smith, and M. P. Murphy, "Antioxidant properties of MitoTEMPOL and its hydroxylamine," Free Radical Research, vol. 43, no. 1, pp. 4-12, 2009.

[24] Q. Ma, S. Chen, Q. Hu et al., "NLRP3 inflammasome contributes to inflammation after intracerebral hemorrhage," Annals of Neurology, vol. 75, no. 2, pp. 209-219, 2014.

[25] P. Gurung, J. R. Lukens, and T.-D. Kanneganti, "Mitochondria: diversity in the regulation of the NLRP3 inflammasome," Trends in Molecular Medicine, vol. 21, no. 3, pp. 193-201, 2015.

[26] F. Bauernfeind, E. Bartok, A. Rieger, L. Franchi, G. Núñez, and V. Hornung, "Cutting edge: reactive oxygen species inhibitors block priming, but not activation, of the NLRP3 inflammasome," Journal of Immunology, vol. 187, no. 2, pp. 613-617, 2011.

[27] S. Mariathasan, D. S. Weiss, K. Newton et al., "Cryopyrin activates the inflammasome in response to toxins and ATP," Nature, vol. 440, no. 7081, pp. 228-232, 2006.

[28] A. Surprenant, F. Rassendren, E. Kawashima, R. A. North, and G. Buell, "The cytolytic P2Z receptor for extracellular ATP identified as a P2X receptor (P2X7)," Science, vol. 272, no. 5262, pp. 735-738, 1996. 
[29] R. Muñoz-Planillo, P. Kuffa, G. Martínez-Colón, B. Smith, T. Rajendiran, and G. Núñez, " $\mathrm{K}^{+}$efflux is the common trigger of NLRP3 inflammasome activation by bacterial toxins and particulate matter," Immunity, vol. 38, no. 6, pp. 1142-1153, 2013.

[30] V. Pétrilli, S. Papin, C. Dostert, A. Mayor, F. Martinon, and J. Tschopp, "Activation of the NALP3 inflammasome is triggered by low intracellular potassium concentration," Cell Death and Differentiation, vol. 14, no. 9, pp. 1583-1589, 2007.

[31] A. Halle, V. Hornung, G. C. Petzold et al., "The NALP3 inflammasome is involved in the innate immune response to amyloid- $\beta$," Nature Immunology, vol. 9, no. 8, pp. 857-865, 2008.

[32] M. Bruchard, G. Mignot, V. Derangère et al., "Chemotherapytriggered cathepsin B release in myeloid-derived suppressor cells activates the Nlrp3 inflammasome and promotes tumor growth," Nature Medicine, vol. 19, no. 1, pp. 57-64, 2013.

[33] C. Dostert, V. Pétrilli, R. Van Bruggen, C. Steele, B. T. Mossman, and J. Tschopp, "Innate immune activation through Nalp3 inflammasome sensing of asbestos and silica," Science, vol. 320, no. 5876, pp. 674-677, 2008.

[34] V. Hornung, F. Bauernfeind, A. Halle et al., "Silica crystals and aluminum salts activate the NALP3 inflammasome through phagosomal destabilization," Nature Immunology, vol. 9, no. 8, pp. 847-856, 2008.

[35] M. Okada, A. Matsuzawa, A. Yoshimura, and H. Ichijo, "The lysosome rupture-activated TAK1-JNK pathway regulates NLRP3 inflammasome activation," Journal of Biological Chemistry, vol. 289, no. 47, pp. 32926-32936, 2014.

[36] T. Murakami, J. Ockinger, J. Yu et al., "Critical role for calcium mobilization in activation of the NLRP3 inflammasome," Proceedings of the National Academy of Sciences of the United States of America, vol. 109, no. 28, pp. 11282-11287, 2012.

[37] G.-S. Lee, N. Subramanian, A. I. Kim et al., "The calciumsensing receptor regulates the NLRP3 inflammasome through $\mathrm{Ca}^{2+}$ and cAMP," Nature, vol. 492, no. 7427, pp. 123-127, 2012.

[38] S. S. Iyer, Q. He, J. R. Janczy et al., "Mitochondrial cardiolipin is required for Nlrp3 inflammasome activation," Immunity, vol. 39, no. 2, pp. 311-323, 2013.

[39] N. Subramanian, K. Natarajan, M. R. Clatworthy, Z. Wang, and R. N. Germain, "The adaptor MAVS promotes NLRP3 mitochondrial localization and inflammasome activation," Cell, vol. 153, no. 2, pp. 348-361, 2013.

[40] S. Park, C. Juliana, S. Hong et al., "The mitochondrial antiviral protein MAVS associates with NLRP3 and regulates its inflammasome activity," Journal of Immunology, vol. 191, no. 8, pp. 4358-4366, 2013.

[41] X. Wang, W. Jiang, Y. Yan et al., "RNA viruses promote activation of the NLRP3 inflammasome through a RIP1-RIP3DRP1 signaling pathway," Nature Immunology, vol. 15, no. 12, pp. 1126-1133, 2014.

[42] K. Nakahira, J. A. Haspel, V. A. K. Rathinam et al., "Autophagy proteins regulate innate immune responses by inhibiting the release of mitochondrial DNA mediated by the NALP3 inflammasome," Nature Immunology, vol. 12, no. 3, pp. 222-230, 2011.

[43] R. van der Burgh, L. Nijhuis, K. Pervolaraki et al., "Defects in mitochondrial clearance predispose human monocytes to interleukin- $1 \beta$ hypersecretion," The Journal of Biological Chemistry, vol. 289, no. 8, pp. 5000-5012, 2014.

[44] J. Harris, M. Hartman, C. Roche et al., "Autophagy controls ILlbeta secretion by targeting pro-IL-1beta for degradation," The Journal of Biological Chemistry, vol. 286, no. 11, pp. 9587-9597, 2011.
[45] S.-Y. Chuang, C.-H. Yang, C.-C. Chou, Y.-P. Chiang, T.-H. Chuang, and L.-C. Hsu, "TLR-induced PAI-2 expression suppresses IL-1 $\beta$ processing èia increasing autophagy and NLRP3 degradation," Proceedings of the National Academy of Sciences of the United States of America, vol. 110, no. 40, pp. 16079-16084, 2013.

[46] C.-S. Shi, K. Shenderov, N.-N. Huang et al., "Activation of autophagy by inflammatory signals limits IL- $1 \beta$ production by targeting ubiquitinated inflammasomes for destruction," Nature Immunology, vol. 13, no. 3, pp. 255-263, 2012.

[47] R. Zhou, A. S. Yazdi, P. Menu, and J. Tschopp, "A role for mitochondria in NLRP3 inflammasome activation," Nature, vol. 469, no. 7329, pp. 221-226, 2011.

[48] B. B. Mishra, V. A. K. Rathinam, G. W. Martens et al., "Nitric oxide controls the immunopathology of tuberculosis by inhibiting NLRP3 inflammasome-dependent processing of IL1ß," Nature Immunology, vol. 14, no. 1, pp. 52-60, 2013.

[49] K. Mao, S. Chen, M. Chen et al., "Nitric oxide suppresses NLRP3 inflammasome activation and protects against LPS-induced septic shock," Cell Research, vol. 23, no. 2, pp. 201-212, 2013.

[50] G. Guarda, M. Braun, F. Staehli et al., "Type I interferon inhibits interleukin-1 production and inflammasome activation," Immunity, vol. 34, no. 2, pp. 213-223, 2011.

[51] G. Pedraza-Alva, L. Pérez-Martínez, L. Valdez-Hernández, K. F. Meza-Sosa, and M. Ando-Kuri, "Negative regulation of the inflammasome: keeping inflammation under control," Immunological Reviews, vol. 265, no. 1, pp. 231-257, 2015.

[52] R. Hanamsagar, V. Torres, and T. Kielian, "Inflammasome activation and IL-1 $\beta /$ IL-18 processing are influenced by distinct pathways in microglia," Journal of Neurochemistry, vol. 119, no. 4, pp. 736-748, 2011.

[53] L. Lammerding, A. Slowik, S. Johann, C. Beyer, and A. Zendedel, "Post-stroke inflammasome expression and regulation in the peri-infarct area by gonadal steroids after transient focal ischemia in the rat brain," Neuroendocrinology, vol. 103, no. 5, pp. 460-475, 2016.

[54] M. Lu, X.-L. Sun, C. Qiao, Y. Liu, J.-H. Ding, and G. Hu, "Uncoupling protein 2 deficiency aggravates astrocytic endoplasmic reticulum stress and nod-like receptor protein 3 inflammasome activation," Neurobiology of Aging, vol. 35, no. 2, pp. 421-430, 2014.

[55] P. Nagyőszi, Á. Nyúl-Tõth, C. Fazakas et al., "Regulation of NOD-like receptors and inflammasome activation in cerebral endothelial cells," Journal of Neurochemistry, vol. 135, no. 3, pp. 551-564, 2015.

[56] D. Lozano, G. S. Gonzales-Portillo, S. Acosta et al., "Neuroinflammatory responses to traumatic brain injury: etiology, clinical consequences, and therapeutic opportunities," Neuropsychiatric Disease and Treatment, vol. 11, pp. 97-106, 2015.

[57] C.-H. Hang, G. Chen, J.-X. Shi, X. Zhang, and J.-S. Li, "Cortical expression of nuclear factor $\kappa \mathrm{B}$ after human brain contusion," Brain Research, vol. 1109, no. 1, pp. 14-21, 2006.

[58] J.-B. Zhao, Y. Zhang, G.-Z. Li, X.-F. Su, and C.-H. Hang, "Activation of JAK2/STAT pathway in cerebral cortex after experimental traumatic brain injury of rats," Neuroscience Letters, vol. 498, no. 2, pp. 147-152, 2011.

[59] I. Yatsiv, M. C. Morganti-Kossmann, D. Perez et al., "Elevated intracranial IL-18 in humans and mice after traumatic brain injury and evidence of neuroprotective effects of IL-18-binding protein after experimental closed head injury," Journal of Cerebral Blood Flow and Metabolism, vol. 22, no. 8, pp. 971-978, 2002. 
[60] H.-D. Liu, W. Li, Z.-R. Chen et al., "Expression of the NLRP3 inflammasome in cerebral cortex after traumatic brain injury in a rat model," Neurochemical Research, vol. 38, no. 10, pp. 20722083, 2013.

[61] J. P. de Rivero Vaccari, G. Lotocki, O. F. Alonso, H. M. Bramlett, W. D. Dietrich, and R. W. Keane, "Therapeutic neutralization of the NLRP1 inflammasome reduces the innate immune response and improves histopathology after traumatic brain injury," Journal of Cerebral Blood Flow and Metabolism, vol. 29, no. 7, pp. 1251-1261, 2009.

[62] S. Adamczak, G. Dale, J. P. de Rivero Vaccari, M. R. Bullock, W. D. Dietrich, and R. W. Keane, "Inflammasome proteins in cerebrospinal fluid of brain-injured patients as biomarkers of functional outcome: clinical article," Journal of Neurosurgery, vol. 117, no. 6, pp. 1119-1125, 2012.

[63] G. R. de Freitas and J. Bogousslavsky, "Primary stroke prevention," European Journal of Neurology, vol. 8, no. 1, pp. 1-15, 2001.

[64] B. R. S. Broughton, D. C. Reutens, and C. G. Sobey, "Apoptotic mechanisms after cerebral ischemia," Stroke, vol. 40, no. 5, pp. e331-e339, 2009.

[65] U. Dirnagl, "Pathobiology of injury after stroke: the neurovascular unit and beyond," Annals of the New York Academy of Sciences, vol. 1268, no. 1, pp. 21-25, 2012.

[66] Q. Alhadidi, M. S. Bin Sayeed, and Z. A. Shah, "Cofilin as a promising therapeutic target for ischemic and hemorrhagic stroke," Translational Stroke Research, vol. 7, no. 1, pp. 33-41, 2016.

[67] H. A. Seifert and K. R. Pennypacker, "Molecular and cellular immune responses to ischemic brain injury," Translational Stroke Research, vol. 5, no. 5, pp. 543-553, 2014.

[68] D. Y.-W. Fann, S.-Y. Lee, S. Manzanero, P. Chunduri, C. G. Sobey, and T. V. Arumugam, "Pathogenesis of acute stroke and the role of inflammasomes," Ageing Research Reviews, vol. 12, no. 4, pp. 941-966, 2013.

[69] D. Y.-W. Fann, S.-Y. Lee, S. Manzanero et al., "Intravenous immunoglobulin suppresses NLRP1 and NLRP3 inflammasome-mediated neuronal death in ischemic stroke," Cell Death and Disease, vol. 4, no. 9, article e790, 2013.

[70] D. Y.-W. Fann, T. Santro, S. Manzanero et al., "Intermittent fasting attenuates inflammasome activity in ischemic stroke," Experimental Neurology, vol. 257, pp. 114-119, 2014.

[71] L. Wang, W. Wang, L. Zhang et al., "Oxygen-glucose deprivation inducing B1 RNA inhibits neuronal cells metabolic activity by NLRP3 and associated proinflammatory cytokines production," Neuroscience Letters, vol. 588, pp. 147-153, 2015.

[72] X. Wang, R. Li, X. Wang, Q. Fu, and S. Ma, "Umbelliferone ameliorates cerebral ischemia-reperfusion injury via upregulating the PPAR gamma expression and suppressing TXNIP/NLRP3 inflammasome," Neuroscience Letters, vol. 600, pp. 182-187, 2015.

[73] Y. Li, J. Li, S. Li et al., "Curcumin attenuates glutamate neurotoxicity in the hippocampus by suppression of ER stress-associated TXNIP/NLRP3 inflammasome activation in a manner dependent on AMPK," Toxicology and Applied Pharmacology, vol. 286, no. 1, pp. 53-63, 2015.

[74] T. Ishrat, I. N. Mohamed, B. Pillai et al., "Thioredoxininteracting protein: a novel target for neuroprotection in experimental thromboembolic stroke in mice," Molecular Neurobiology, vol. 51, no. 2, pp. 766-778, 2015.

[75] M. Ito, T. Shichita, M. Okada et al., “Bruton's tyrosine kinase is essential for NLRP3 inflammasome activation and contributes to ischaemic brain injury," Nature Communications, vol. 6, article 7360, 2015.

[76] J. Zhao, Y. Mou, J. D. Bernstock et al., "Synthetic oligodeoxynucleotides containing multiple telemeric TTAGGG motifs suppress inflammasome activity in macrophages subjected to oxygen and glucose deprivation and reduce ischemic brain injury in stroke-prone spontaneously hypertensive rats," PLoS ONE, vol. 10, no. 10, Article ID e0140772, 2015.

[77] R. F. Keep, Y. Hua, and G. Xi, "Intracerebral haemorrhage: mechanisms of injury and therapeutic targets," The Lancet Neurology, vol. 11, no. 8, pp. 720-731, 2012.

[78] K. Kang, Y.-J. Kim, Y.-H. Kim et al., "Lithium pretreatment reduces brain injury after intracerebral hemorrhage in rats," Neurological Research, vol. 34, no. 5, pp. 447-454, 2012.

[79] B. Lei, H. N. Dawson, B. Roulhac-Wilson, H. Wang, D. T. Laskowitz, and M. L. James, “Tumor necrosis factor alpha antagonism improves neurological recovery in murine intracerebral hemorrhage," Journal of Neuroinflammation, vol. 10, article 103, 2013.

[80] A. S. Pandey and G. Xi, "Intracerebral hemorrhage: a multimodality approach to improving outcome," Translational Stroke Research, vol. 5, no. 3, pp. 313-315, 2014.

[81] S. Chen, Q. Yang, G. Chen, and J. H. Zhang, "An update on inflammation in the acute phase of intracerebral hemorrhage," Translational Stroke Research, vol. 6, no. 1, pp. 4-8, 2014.

[82] X.-Y. Xiong and Q.-W. Yang, "Rethinking the roles of inflammation in the intracerebral hemorrhage," Translational Stroke Research, vol. 6, no. 5, pp. 339-341, 2015.

[83] L. Feng, Y. Chen, R. Ding et al., "P2X7R blockade prevents NLRP3 inflammasome activation and brain injury in a rat model of intracerebral hemorrhage: involvement of peroxynitrite," Journal of Neuroinflammation, vol. 12, no. 1, article 190, 2015.

[84] B. Yuan, H. Shen, L. Lin, T. Su, S. Zhong, and Z. Yang, "Recombinant adenovirus encoding NLRP3 RNAi attenuate inflammation and brain injury after intracerebral hemorrhage," Journal of Neuroimmunology, vol. 287, pp. 71-75, 2015.

[85] Z. Yang, L. Zhong, R. Xian, and B. Yuan, "MicroRNA223 regulates inflammation and brain injury via feedback to NLRP3 inflammasome after intracerebral hemorrhage," Molecular Immunology, vol. 65, no. 2, pp. 267-276, 2015.

[86] J. van Gijn, R. S. Kerr, and G. J. Rinkel, "Subarachnoid haemorrhage," The Lancet, vol. 369, no. 9558, pp. 306-318, 2007.

[87] N. Etminan, "Aneurysmal subarachnoid hemorrhage-status quo and perspective," Translational Stroke Research, vol. 6, no. 3, pp. 167-170, 2015.

[88] F. A. Sehba, J. Hou, R. M. Pluta, and J. H. Zhang, "The importance of early brain injury after subarachnoid hemorrhage," Progress in Neurobiology, vol. 97, no. 1, pp. 14-37, 2012.

[89] B. Caner, J. Hou, O. Altay, M. Fuj II, and J. H. Zhang, "Transition of research focus from vasospasm to early brain injury after subarachnoid hemorrhage," Journal of Neurochemistry, vol. 123, supplement 2, pp. 12-21, 2012.

[90] M. K. Tso and R. L. Macdonald, "Subarachnoid hemorrhage: a review of experimental studies on the microcirculation and the neurovascular unit," Translational Stroke Research, vol. 5, no. 2, pp. 174-189, 2014.

[91] H. Suzuki, "What is early brain injury?" Translational stroke research, vol. 6, no. 1, pp. 1-3, 2015.

[92] S. Chen, H. Feng, P. Sherchan et al., "Controversies and evolving new mechanisms in subarachnoid hemorrhage," Progress in Neurobiology, vol. 115, pp. 64-91, 2014. 
[93] J. Li, J. Chen, H. Mo et al., "Minocycline protects against NLRP3 inflammasome-induced inflammation and P53associated apoptosis in early brain injury after subarachnoid hemorrhage," Molecular Neurobiology, vol. 53, no. 4, pp. 2668-2678, 2016.

[94] A. Shao, H. Wu, Y. Hong et al., "Hydrogen-rich saline attenuated subarachnoid hemorrhage-induced early brain injury in rats by suppressing inflammatory response: possible involvement of NF- $\kappa$ B pathway and NLRP3 inflammasome," Molecular Neurobiology, vol. 53, no. 5, pp. 3462-3476, 2016.

[95] Y. Dong, C. Fan, W. Hu et al., "Melatonin attenuated early brain injury induced by subarachnoid hemorrhage via regulating NLRP3 inflammasome and apoptosis signaling," Journal of Pineal Research, vol. 60, no. 3, pp. 253-262, 2016.

[96] M. Terlizzi, V. Casolaro, A. Pinto, and R. Sorrentino, "Inflammasome: cancer's friend or foe?" Pharmacology and Therapeutics, vol. 143, no. 1, pp. 24-33, 2014.

[97] M. T. Chow, J. Sceneay, C. Paget et al., "NLRP3 suppresses NK cell-mediated responses to carcinogen-induced tumors and metastases," Cancer Research, vol. 72, no. 22, pp. 5721-5732, 2012.

[98] L.-C. Chen, L.-J. Wang, N.-M. Tsang et al., "Tumour inflammasome-derived IL- $1 \beta$ recruits neutrophils and improves local recurrence-free survival in EBV-induced nasopharyngeal carcinoma," EMBO Molecular Medicine, vol. 4, no. 12, pp. 1276-1293, 2012.

[99] L. Tarassishin, A. Bauman, H.-S. Suh, and S. C. Lee, "Antiviral and anti-inflammatory mechanisms of the innate immune transcription factor interferon regulatory factor 3: relevance to human CNS diseases," Journal of Neuroimmune Pharmacology, vol. 8, no. 1, pp. 132-144, 2013.

[100] I. Yang, S. J. Han, G. Kaur, C. Crane, and A. T. Parsa, “The role of microglia in central nervous system immunity and glioma immunology," Journal of Clinical Neuroscience, vol. 17, no. 1, pp. 6-10, 2010.

[101] L. Zitvogel, O. Kepp, L. Galluzzi, and G. Kroemer, "Inflammasomes in carcinogenesis and anticancer immune responses," Nature Immunology, vol. 13, no. 4, pp. 343-351, 2012.

[102] L. Tarassishin, D. Casper, and S. C. Lee, "Aberrant expression of interleukin- $1 \beta$ and inflammasome activation in human malignant gliomas," PLoS ONE, vol. 9, no. 7, Article ID e103432, 2014.

[103] N. Murphy, T. R. Cowley, J. C. Richardson et al., “The neuroprotective effect of a specific $\mathrm{P}_{2} \mathrm{X}_{7}$ receptor antagonist derives from its ability to inhibit assembly of the NLRP3 inflammasome in glial cells," Brain Pathology, vol. 22, no. 3, pp. 295-306, 2012.

[104] L. Li and Y. Liu, "Aging-related gene signature regulated by Nlrp3 predicts glioma progression," American Journal of Cancer Research, vol. 5, no. 1, pp. 442-449, 2015.

[105] M. T. Heneka, M. P. Kummer, and E. Latz, "Innate immune activation in neurodegenerative disease," Nature Reviews Immunology, vol. 14, no. 7, pp. 463-477, 2014.

[106] P. Bossù, A. Ciaramella, M. L. Moro et al., "Interleukin 18 gene polymorphisms predict risk and outcome of Alzheimer's disease," Journal of Neurology, Neurosurgery and Psychiatry, vol. 78, no. 8, pp. 807-811, 2007.

[107] J. Ojala, I. Alafuzoff, S.-K. Herukka, T. van Groen, H. Tanila, and T. Pirttilä, "Expression of interleukin-18 is increased in the brains of Alzheimer's disease patients," Neurobiology of Aging, vol. 30, no. 2, pp. 198-209, 2009.

[108] C. L. Masters and D. J. Selkoe, "Biochemistry of amyloid $\beta$-protein and amyloid deposits in Alzheimer disease," Cold
Spring Harbor Perspectives in Medicine, vol. 2, no. 6, Article ID a006262, 2012.

[109] M.-S. Tan, J.-T. Yu, T. Jiang, X.-C. Zhu, and L. Tan, “The NLRP3 inflammasome in Alzheimer's disease," Molecular Neurobiology, vol. 48, no. 3, pp. 875-882, 2013.

[110] H. Braak, K. Del Tredici, U. Rüb, R. A. I. De Vos, E. N. H. Jansen Steur, and E. Braak, "Staging of brain pathology related to sporadic Parkinson's disease," Neurobiology of Aging, vol. 24, no. 2, pp. 197-211, 2003.

[111] Y. Zhou, M. Lu, R. H. Du et al., "MicroRNA-7 targets Nod-like receptor protein 3 inflammasome to modulate neuroinflammation in the pathogenesis of Parkinson's disease," Molecular Neurodegeneration, vol. 11, article 28, 2016.

[112] G. Codolo, N. Plotegher, T. Pozzobon et al., "Triggering of inflammasome by aggregated $\alpha$-synuclein, an inflammatory response in synucleinopathies," PLoS ONE, vol. 8, no. 1, Article ID e55375, 2013.

[113] C. K. Glass, K. Saijo, B. Winner, M. C. Marchetto, and F. H. Gage, "Mechanisms underlying inflammation in neurodegeneration," Cell, vol. 140, no. 6, pp. 918-934, 2010.

[114] A. Vezzani, J. French, T. Bartfai, and T. Z. Baram, "The role of inflammation in epilepsy," Nature Reviews Neurology, vol. 7, no. 1, pp. 31-40, 2011.

[115] X.-F. Meng, L. Tan, M.-S. Tan et al., "Inhibition of the NLRP3 inflammasome provides neuroprotection in rats following amygdala kindling-induced status epilepticus," Journal of Neuroinflammation, vol. 11, no. 1, article 212, 2014.

[116] W. J. Streit, "Microglia as neuroprotective, immunocompetent cells of the CNS," Glia, vol. 40, no. 2, pp. 133-139, 2002.

[117] Y. Jamilloux, R. Pierini, M. Querenet et al., "Inflammasome activation restricts Legionella pneumophila replication in primary microglial cells through flagellin detection," Glia, vol. 61, no. 4, pp. 539-549, 2013.

[118] T. Hoegen, N. Tremel, M. Klein et al., "The NLRP3 inflammasome contributes to brain injury in pneumococcal meningitis and is activated through ATP-dependent lysosomal cathepsin B release," The Journal of Immunology, vol. 187, no. 10, pp. 54405451, 2011.

[119] M. Yu, K. Zhang, W. Qi et al., "Expression pattern of NLRP3 and its related cytokines in the lung and brain of avian influenza virus H9N2 infected BALB/c mice," Virology Journal, vol. 11, article 229, 2014.

[120] F. Shi, M. Kouadir, and Y. Yang, "NALP3 inflammasome activation in protein misfolding diseases," Life Sciences, vol. 135, no. 1, pp. 9-14, 2015.

[121] M. Inoue, K. L. Williams, M. D. Gunn, and M. L. Shinohara, "NLRP3 inflammasome induces chemotactic immune cell migration to the CNS in experimental autoimmune encephalomyelitis," Proceedings of the National Academy of Sciences of the United States of America, vol. 109, no. 26, pp. 10480-10485, 2012. 


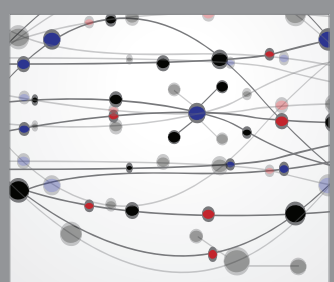

The Scientific World Journal
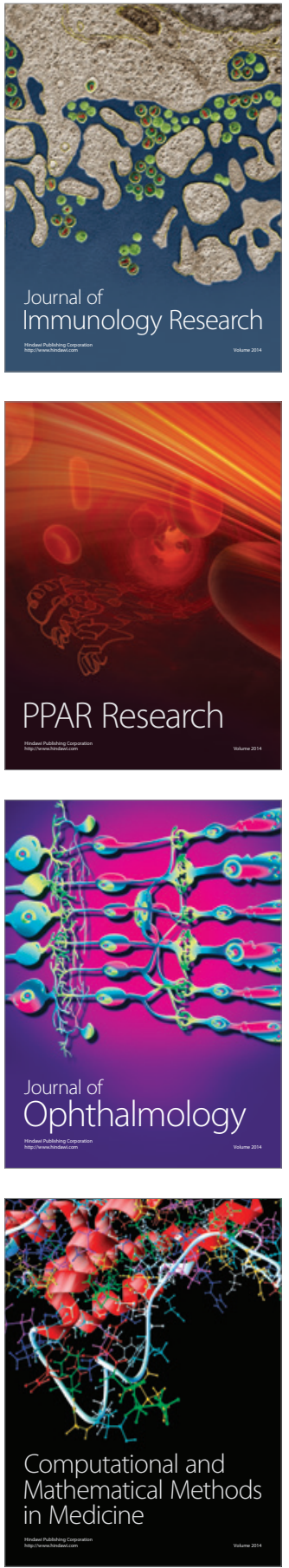

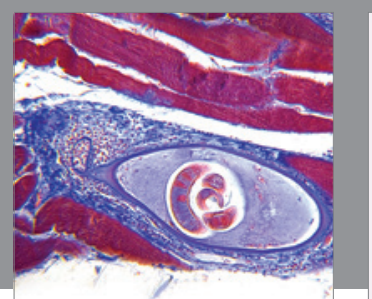

Gastroenterology Research and Practice

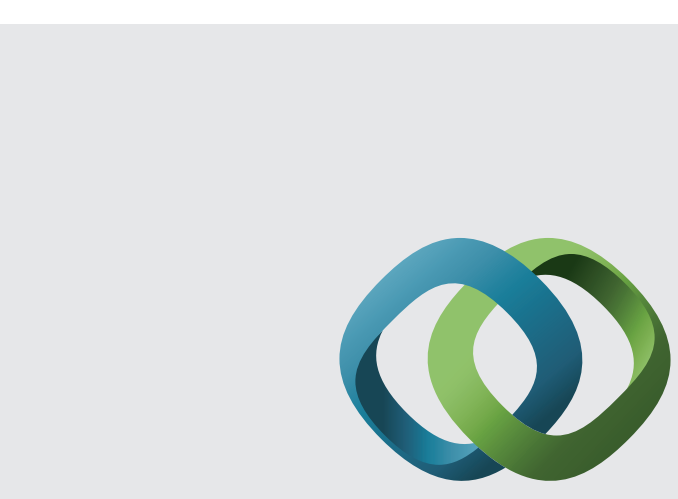

\section{Hindawi}

Submit your manuscripts at

http://www.hindawi.com
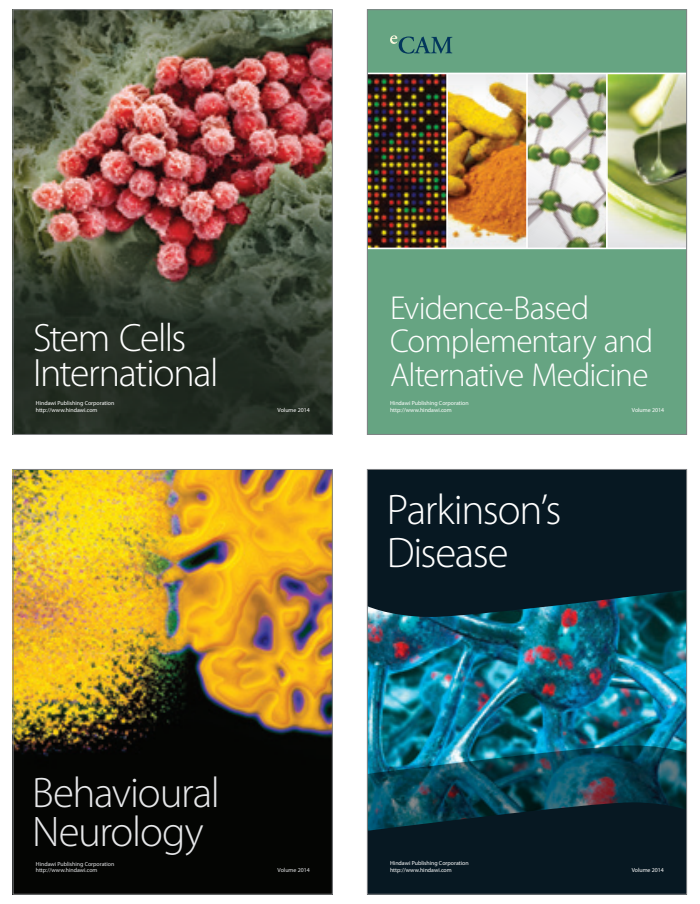
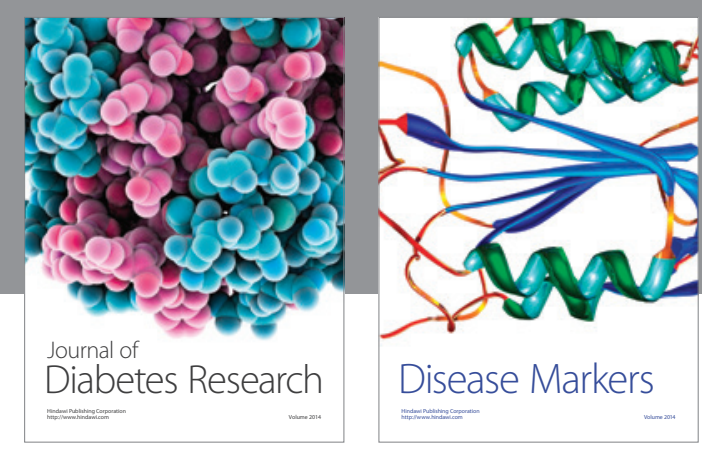

Disease Markers
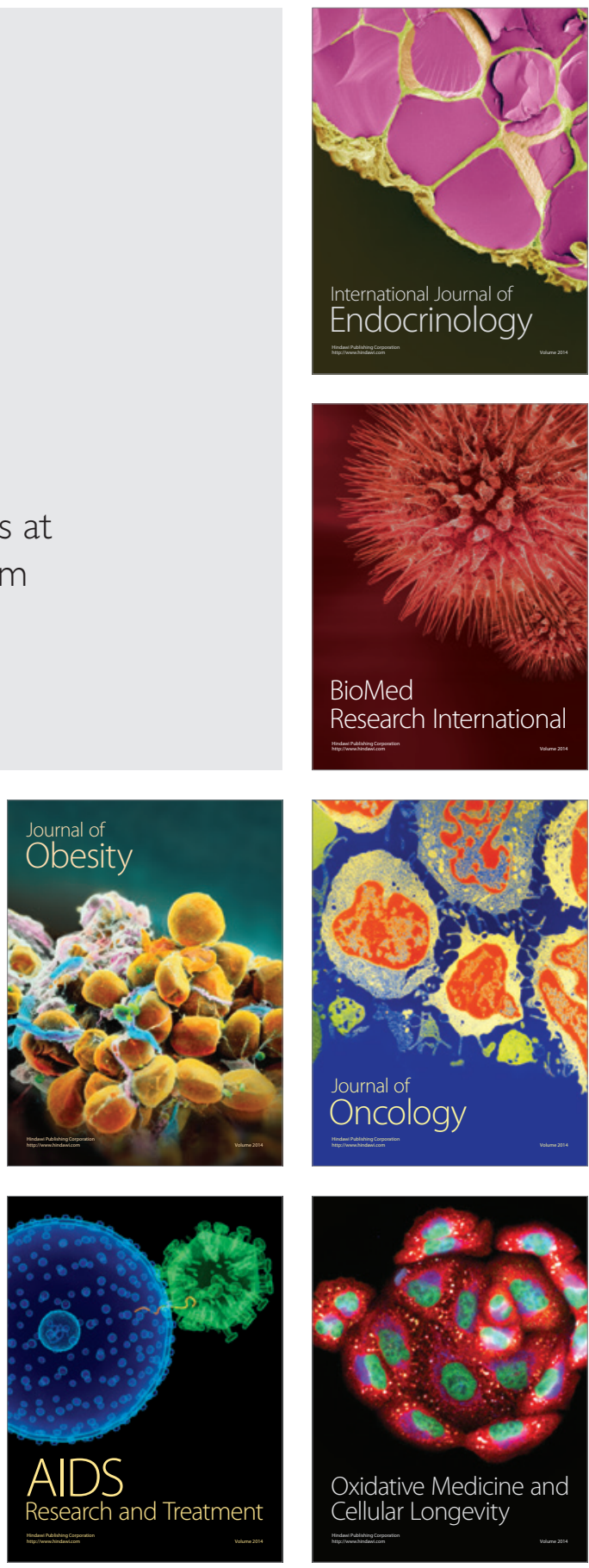